\title{
Neutrophil CD64 Expression: A Sensitive Diagnostic Marker for Late-Onset Nosocomial Infection in Very Low Birthweight Infants
}

\author{
PAK C. NG, KAREN LI, RAYMOND P. O. WONG, KIT M. CHUI, ERIC WONG, AND TAI F. FOK \\ Department of Pediatrics [P.C.N., K.L., R.P.O.W., K.M.C., T.F.F.] and Center of Clinical Trials and \\ Epidemiological Research [E.W.], Prince of Wales Hospital, Chinese University of Hong Kong, \\ P.R. China
}

\begin{abstract}
This study aims to evaluate the diagnostic utilities of four leukocyte surface antigens - two lymphocyte antigens (CD25 and CD45RO) and two neutrophil antigens (CD11b and CD64) - for identification of late-onset nosocomial bacterial infection in preterm, very low birthweight infants, and to define the optimal cutoff value for each marker so that it may act as a reference with which future studies can be compared. Very low birthweight infants in whom infection was suspected when they were $>72 \mathrm{~h}$ of age were eligible for the study. A full sepsis screen was performed in each episode. IL-6, C-reactive protein, and leukocyte surface antigens (CD25, CD45RO, CD11b, and CD64) were measured at 0 (at the time of sepsis evaluation), 24, and $48 \mathrm{~h}$ by standard biochemical methods and quantitative flow cytometric analysis. The diagnostic utilities including sensitivity, specificity, and positive and negative predictive values of each marker and combination of markers for predicting late-onset neonatal infection were determined. One hundred twenty-seven episodes of suspected clinical sepsis were investigated in 80 infants. Thirty-seven episodes were proven infection. The calculated optimal cutoff values for CD25, CD45RO, CD11b, and CD64 were $3,100,2,900,10,450$, and 4,000 phycoerythrinmolecules bound per cell, respectively. An interim analysis of data after 68 episodes suggested that CD25 and CD45RO were poor predictors of neonatal infection with sensitivity or specificity $<75 \%$ during a single measurement. Thus, these two markers were excluded from further investigation. In the final analysis, CD64 has the highest sensitivity (95-97\%) and negative predic-
\end{abstract}

\section{ABSTRACT}

tive value (97-99\%) at 0 and $24 \mathrm{~h}$ after the onset. The addition of IL-6 or C-reactive protein $(0 \mathrm{~h})$ to CD64 (24 h) further enhanced the sensitivity and negative predictive value to $100 \%$, and has the specificity and positive predictive value exceeding $88 \%$ and $80 \%$, respectively. Neutrophil CD64 expression is a very sensitive marker for diagnosing late-onset nosocomial infection in very low birthweight infants. If further validated, the use of CD64 as an infection marker should allow early discontinuation of antibiotic treatment at $24 \mathrm{~h}$ without waiting for the definitive microbiologic culture results. The quantitative flow cytometric analysis applied in this study could be developed into a routine clinical test with high comparability and reproducibility across different laboratories. (Pediatr Res 51: 296-303, 2002)

CRP, C-reactive protein

\section{Abbreviations}

CSF, cerebrospinal fluid

Fc $\gamma$ RI, Fc $\gamma$-receptor I

MFI, mean fluorescence intensity

NEC, necrotizing enterocolitis

NPV, negative predictive value

perCP, peridinin chlorophyll protein

PE, phycoerythrin

PPV, positive predictive value

ROC, receiver operating characteristics

TNF- $\alpha$, tumor necrosis factor- $\alpha$

VLBW, very low birthweight
Late-onset nosocomial bacterial infection is an important cause of morbidity and mortality in newborn infants requiring intensive care (1). Preterm VLBW infants are particularly vulnerable because of immune immaturity, severe underlying conditions, and frequent requirement of invasive procedures such as arterial or venous umbilical catheterization, central

Received May 9, 2001; accepted October 23, 2001

Correspondence: P.C. Ng, Department of Pediatrics, Level 6, Clinical Sciences Building, Prince of Wales Hospital, Shatin, N.T., Hong Kong; e-mail: pakcheungng@ cuhk.edu.hk venous line placement, and insertion of chest drains. It has been estimated that up to $25 \%$ of VLBW infants are affected by nosocomial infection during their stay in neonatal intensive care (1). Despite the development of new broad-spectrum antibiotics and advanced life support treatment, a significant proportion of infected infants still follows a rapid downhill course leading to septicemic shock, disseminated intravascular coagulation, and death within hours of deterioration. The overall statistics indicate that infection in the newborn period is associated with approximately $10 \%$ of cases of neonatal death 
(2). Thus, a reliable infection marker or a set of markers are required to promptly and accurately identify the infected cases so that treatment can be started without delay. Equally difficult is the exclusion of infection in infants with suspected sepsis, as continuation of broad-spectrum antibiotics for presumptive bacterial infection frequently leads to unnecessary treatment and also the possibility of emergence of multiresistant organisms. In the past decade, many investigators, including our own investigating team, have used various hematologic and biochemical markers, and cytokines such as immature/total neutrophil ratio $(3,4)$, CRP $(4-8)$, procalcitonin $(8-10)$, TNF- $\alpha$ $(5,11)$, IL-1 $\beta$ (5), soluble IL-1ra (12), IL-2 receptor (13), IL-6 $(5,12)$, IL-8 $(7,14)$, IL-10 (15), and markers of complementactivation $(16,17)$ as indicators for early or confirmation of diagnosis of neonatal infection. We have previously demonstrated that the combination of IL- 6 and serial CRP measurements gave a reasonably high sensitivity and specificity for diagnosing late-onset nosocomial infection (5). However, the use of IL-6 remains principally as a research tool and has not gained acceptance for clinical application because most laboratories would be inclined to perform this expensive test in batches and, thus, defeats the purpose of using it as an early warning marker (5). Recently, advances in flow cytometric technology have paved the way to easy detection of cellsurface antigens on blood cells. Specific cell-surface antigens are expressed in large quantities after the leukocytes are activated by bacteria or toxins $(18,19)$. Multicolor flow cytometric analysis also has the advantage over immunoassay of being able to localize the activated markers on a specific cell type, and the test can be performed on an ad hoc basis requiring only minimal volume of blood sample ( $0.05 \mathrm{~mL}$ of whole blood).

This study selected four specific cell-surface activation markers, two lymphocyte markers (CD25 and CD45RO) and two neutrophil markers (CD11b and CD64), for assessing their abilities and precision in identifying late-onset bacterial infection in VLBW infants. The choice of these markers has been purposefully chosen. All have been indicated to be upregulated during an infective episode or when they come into contact with microbial products (18-23). The aims of this study were 1) to delineate the pattern of expression of these cell-surface antigens during bacterial infection and in relation to successful treatment; 2) to establish the diagnostic utilities (sensitivity, specificity, and positive and negative predictive values) of these markers, both individually and in combination, for early diagnosis of late-onset neonatal systemic infection in VLBW infants; and 3) to define the optimal cutoff value for each marker using the ROC so that it may act as a reference with which future studies can be compared.

\section{METHODS}

Patients. Preterm infants, with 1) birth weight $<1500$ g, 2) postnatal age $>72 \mathrm{~h}, 3$ ) signs and symptoms suggestive of systemic infection and requiring full sepsis evaluation and antibiotic treatment, and 4) parental consent, in the neonatal unit at Prince of Wales Hospital, Hong Kong, were eligible for enrollment into the study. Patients who were already receiving parenteral antibiotic at the time of sepsis evaluation, or have severe congenital or chromosomal abnormalities, were excluded. Recruitment of suspected infection episode was conducted prospectively during a 22-mo period.

Methods. Signs and symptoms suggestive of clinical sepsis have been described in detail in our previous study (5). All infants were recruited at the time of evaluation for suspected clinical sepsis. In each episode, a full sepsis screen was performed, which included CSF, blood, urine, stool, and endotracheal aspirate (infants on respirator) cultures for bacteria and fungi; removal and culture of indwelling central lines or catheters; and cultures of specific sites and surgical specimens such as peritoneal fluid, abscess, and biopsy specimen. Chest radiograph was routinely performed during the initial screening procedure, and an abdominal radiograph would be requested when patients presented with signs suggestive of intraabdominal disease. Hematologic and biochemical laboratory investigations including a complete blood cell, differential white cell, and platelet counts; arterial blood gas; and serum glucose concentration were also performed. In addition to our routine serial CRP measurements, blood specimens for IL-6 $(0.3 \mathrm{~mL}$ heparinized blood), lymphocyte cell-surface antigens CD25 and CD45RO, and neutrophil cell-surface antigens CD11b and CD64 (0.3 mL EDTA blood) were obtained for evaluation. The first sample was taken at the time of the initial sepsis evaluation $(0 \mathrm{~h})$, and two further samples were obtained at 24 and $48 \mathrm{~h}$ after the onset for monitoring the clinical progress and response to antibiotic treatment. This schedule of blood sampling coincided exactly with our unit policy for serial blood count and CRP measurements after a suspected episode of infection had been identified. Intravenous broad-spectrum antibiotics were started immediately after the sepsis screening and the first set of blood sample had been performed. Moreover, we followed the same classification of infection as in our previous study (5). Three categories of infective episodes were prospectively defined. Briefly, they were as follows: group 1, the infected group, which consisted of episodes that had been confirmed as septicemia, meningitis, pneumonia, peritonitis, systemic fungal infection, or NEC (stage II or above in Bell's classification) (24). Group 2 was defined as the noninfected group and consisted of episodes that met the screening criteria for suspected clinical sepsis but were subsequently proven not to be infected. Group 3 was the control group and consisted of blood samples taken once from 20 well, VLBW infants between wk 1 and 5 of postnatal age for IL-6, CRP, and cellsurface antigen measurements. The collection of the latter blood samples coincided with the weekly monitoring of $\mathrm{Hb}$, liver function, and bone profile of preterm infants.

Measurement of biochemical and cell-surface markers. Blood samples collected from indwelling arterial lines or venipunctures were immersed in ice and immediately transported to the laboratory for processing. Plasma was separated by centrifugation $(1900 \times g$ for $5 \mathrm{~min})$ at $4^{\circ} \mathrm{C}$ and stored in $200-\mu \mathrm{L}$ aliquots at $-80^{\circ} \mathrm{C}$ until analysis. CRP was measured by a turbidity assay against control standards, as specified by the manufacturer (Behring Diagnostics Inc., Westwood, MA, U.S.A.). IL-6 was measured by the ELISA technique using a commercially available kit (R\&D System Inc., Minneapolis, 
MN, U.S.A.). The inter- and intraassay variabilities were within the 5\% limits specified by the manufacturers.

Quantitative flow cytometric analysis of the EDTA specimens was performed by the FACSCalibur machine (Becton Dickinson Immunocytometry Systems, San Jose, CA, U.S.A.). The cytometer was routinely optimized using the CaliBRITE beads (Becton Dickinson Immunocytometry Systems). Before each analysis, the QuantiBRITE PE beads (Becton Dickinson Immunocytometry Systems) conjugated with four predefined levels of PE molecules were used to construct a standard linear regression curve. The amount of PE molecule bound per cell stands for the absolute number of fluorochrome antibody binding sites per cell. In principle, each QuantiBRITE grade antibody molecule is conjugated to one PE fluorochrome molecule. The instrument measures the MFI on each cell. By comparing the MFI of the cell with those of the four QuantiBRITE beads, of which the number of fluorochrome per bead is known, we can determine the number of antibody binding sites on the cell using the QuantiQuest computer software (Becton Dickinson Immunocytometry Systems). This method represents an improvement over the relative fluorescent intensity method (MFI measurement), which may be affected by subtle, day-to-day operational and instrumental fluctuations. Thus, this technique provides an accurate and quantitative comparison of the cellsurface antigen expression in time serial analysis $(25,26)$. Once the method of sample collection and analytic strategies are standardized, these data can be used for interlaboratory comparison.

Freshly collected EDTA blood was maintained at $4{ }^{\circ} \mathrm{C}$ and stained within $15 \mathrm{~min}$ after arrival at the laboratory using FITC-, PE- ,or perCP- conjugated antibodies (Becton Dickinson Immunocytometry Systems). The CD64 antibody was of QuantiBRITE grade. The EDTA blood samples were stained with $10 \mu \mathrm{L}$ of each antibody in the following tubes: CD16FITC/CD11b-PE/CD45-perCP, CD3-FITC/CD25-PE/CD45perCP, CD3-FITC/CD45RO-PE/CD45-perCP, CD64-PE/ $\mathrm{CD} 45$-perCP, and the respective isotypic controls. Except for the CD64 measurement, all tubes were incubated in the dark for $20 \mathrm{~min}$ at room temperature before red blood cells were lysed for $10 \mathrm{~min}$ in the FACS Lysing Solution (Becton Dickinson Immunocytometry Systems). After washing with $3 \mathrm{~mL}$ of PBS, these cells were resuspended in 1\% paraformaldehyde, and flow cytometric analysis was immediately performed. For the CD64 antibody staining, the lyse-no-wash method was used as recommended by the manufacturer. The samples were incubated at room temperature for $1 \mathrm{~h}$, and then the red blood cells were lysed by the FACS Lysing Solution for a further hour. Thirty thousand events were acquired for each tube, and the CellQuest software (Becton Dickinson Immunocytometry Systems) was used for quantitative analysis of fluorescence. The three parts differential populations (lymphocytes, monocytes, and granulocytes) were identified and gated by their CD45/side-scatter profile. For CD11b staining, the neutrophils were further characterized by their positive CD16 expression, which distinguished them from the $\mathrm{CD} 16^{-}$eosinophils and basophils. The expression (geometric mean) of CD64 and $\mathrm{CD} 11 \mathrm{~b}$ on neutrophils and CD45RO and CD25 on T lymphocytes $\left(\mathrm{CD} 45^{+} \mathrm{CD}^{+}\right.$cells) was quantitatively measured.
Interim analysis. As none of the cell-surface antigens have been systematically and quantitatively evaluated for diagnosis of systemic infection in preterm infants, an interim analysis of the data was planned after approximately half of the suspected episodes had been studied. Cell-surface markers with sensitivity and specificity consistently less than $75 \%$ were considered unsatisfactory and were excluded from further investigation.

Statistical analysis. The demographic data of the three groups, and the levels of infection markers among the infected (group 1; $0 \mathrm{~h}$ ), noninfected (group 2; $0 \mathrm{~h}$ ), and control group (group 3) were compared using the Kruskal-Wallis test and $\chi^{2}$ test. In addition, mixed-effects models (27) were used for comparing the longitudinal measurements of different markers between the infected and noninfected groups. The statistical analysis was performed on raw or logarithmically transformed data as appropriate.

As there were no recommended diagnostic cutoff values for any of the cell-surface antigens in preterm neonates, an ROC curve was constructed for each sampling time point for each marker. The ROC graph with the maximum area under the curve was chosen, and the optimal cutoff value for individual markers was then determined on the graph by minimizing the number of misclassified episodes. As the diagnostic marker should ideally identify all genuinely infected episodes (i.e. $100 \%$ sensitivity) and at the same time would not misclassify too many noninfected cases (i.e. a high specificity), the optimal cutoff value was, therefore, chosen with the sensitivity approaching $100 \%$ and specificity $>85 \%$. However, if the diagnostic marker was unable to satisfy the above criteria, the optimal cutoff value would be chosen so that both the sensitivity and specificity approached $75 \%$. The calculated optimal cutoff values enabled us to work out the diagnostic utilities: sensitivity, specificity, and positive and negative predictive values of the cell-surface antigens, and also to select the best marker or combination of markers at the most appropriate sampling time for diagnosing late-onset nosocomial infection in VLBW infants. A combination of tests was considered positive if any one of the selected markers exceeded their respective cutoff values. In addition, the optimal cutoff values for IL- 6 and CRP have already been determined by the ROC method in our previous study, and they were $31 \mathrm{pg} / \mathrm{mL}$ and 12 $\mathrm{mg} / \mathrm{L}$, respectively (5).

All statistical tests were performed by SPSS for Windows (Release 10, SPSS Inc., Chicago, IL, U.S.A.) and MIXREG computer software (28), which used the maximum marginal likelihood, expectation and maximization algorithm, and Fisher scoring solution. The level of significance was set at 5\% in all comparisons.

Ethical approval. The study was approved by the Research Ethics Committee of the Chinese University of Hong Kong. Informed consent was obtained from the parents or guardians for all study patients.

\section{RESULTS}

Interim analysis. An interim analysis was performed after 68 episodes of suspected clinical sepsis have been investigated. Eighteen of the 68 episodes were identified to be genuine 
infection. CD25 expression with a calculated optimal cutoff value of $3100 \mathrm{PE}$-molecules bound/cell was found to have sensitivities, specificities, and positive and negative predictive values ranging from 50 to $75 \%, 34$ to $43 \%, 21$ to $27 \%$, and 65 to $81 \%$, respectively, during the three specific times (i.e. 0,24 , and $48 \mathrm{~h}$ ) of sepsis evaluation. Similarly, CD45RO expression with a calculated optimal cutoff value of 2900 PE-molecules bound/cell was found to have sensitivities, specificities, and positive and negative predictive values ranging from 65 to $83 \%, 50$ to $100 \%, 43$ to $100 \%$, and 82 to $87 \%$, respectively. As both markers did not satisfy the predetermined criteria of having the sensitivity and specificity $>75 \%$ during a single measurement, they were considered unreliable for predicting infection in VLBW infants. Hence, these two cell-surface markers were excluded from further investigation.

Final analysis. The clinical characteristics of the three study groups are summarized in Table 1. There were no significant differences in gestational age, birthweight, Apgar scores at 1 and $5 \mathrm{~min}$, and male and female ratio among the groups.

One hundred twenty-seven episodes of suspected clinical sepsis were investigated in 80 VLBW infants. One, one, seven, and 26 patients had sepsis screen performed five, four, three, and two times, respectively. The remaining 45 infants received only one sepsis screen. Thirty-seven of the 127 episodes of suspected clinical sepsis were confirmed infection and NEC. Detailed accounts of the clinical characteristics and organisms are summarized in Table 2 .

Figure 1 shows the flow cytometric analysis of CD64 expression on neutrophils in a typical infected infant and in a noninfected infant. In episodes of confirmed infection, both IL- 6 and CD $11 \mathrm{~b}$ manifested their peak concentrations at the time of evaluation for suspected clinical sepsis $(0 \mathrm{~h})$, whereas CD64 levels were noted to be markedly raised at both 0 and $24 \mathrm{~h}$, and the maximum CRP concentration peaked approximately $24 \mathrm{~h}$ after the onset (Fig. 2). Table 3 illustrates the levels of the biochemical markers and the neutrophil cellsurface antigen expression at the onset $(0 \mathrm{~h})$ of sepsis evaluation. The postnatal age of blood sampling did not differ significantly among the three groups. All markers in the infected group were significantly increased when compared with the corresponding values of the noninfected group at 0 and $24 \mathrm{~h}(p$ $<0.05)$. Likewise, the levels of markers in the infected group at $0 \mathrm{~h}$ were also significantly higher than those of the control group $(p<0.0001$, Table 3$)$. In contrast, no significant difference was detected between the levels of the noninfected group at $0 \mathrm{~h}$ and the control group (Table 3).
Table 4 summarizes the diagnostic utilities of IL-6, CRP, and the neutrophil surface antigens CD11b and CD64, using the cutoff values (IL-6, $31 \mathrm{pg} / \mathrm{mL}$; CRP, $12 \mathrm{mg} / \mathrm{L}$ ) determined in our previous study (5), and the calculated optimal cutoff values (CD11b, 10450 PE-molecules bound/cell; CD64, 4000 PE-molecules bound/cell) derived by minimizing the number of misclassified episodes over all possible cutoff values for 0 , 24, and $48 \mathrm{~h}$. The ROC curves of CD11b and CD64 at 0 and $24 \mathrm{~h}$ are shown in Figure 3. The assessment of individual markers indicated that CD64 has the highest sensitivity and negative predictive value at 0 and $24 \mathrm{~h}$. Its specificity and positive predictive value also exceeded $88 \%$ and $80 \%$, respectively, during the study period (Table 4). Both IL-6 and CD11b have the highest sensitivity at the beginning of sepsis evaluation $(0 \mathrm{~h}$ ), whereas CRP, which peaked at $24 \mathrm{~h}$ (Fig. 2), was found to be a highly specific marker for predicting nosocomial infection (Table 4).

In addition, a comparison of the diagnostic utilities of the three best markers, CD64, IL-6, and CRP in combination versus individual markers, suggested that the use of multiple markers was associated with marginally higher sensitivity and negative predictive value (Tables 4 and 5). After calculating all possible combinations and permutations for the best three markers within the first $48 \mathrm{~h}$, IL- 6 or CRP at $0 \mathrm{~h}$ combined with CD64 at $24 \mathrm{~h}$ provided the best overall combination, with sensitivity and specificity reaching $100 \%$ and $86 \%$, respectively. In contrast, the addition of CRP at 24 or $48 \mathrm{~h}$ to CD64 (24 h) might further enhance the specificity and positive predictive value, but has the disadvantage of lowering the sensitivity and negative predictive value (Table 5). Table 5 summarizes the permutations of the best three markers with the highest sensitivity and negative predictive value.

\section{DISCUSSION}

$\mathrm{CD} 11 \mathrm{~b}$ is an $\alpha$ subunit of the $\beta_{2}$ integrin adhesion molecule. It is a cell-surface antigen of neutrophil and is normally expressed at a very low level on the surface of nonactivated cells $(18,19)$. Its expression on neutrophil cell surface, however, increases substantially within a few minutes after the cell comes into contact with bacteria or endotoxins $(29,30)$. This unique property enables CD11b to be used as a potential early warning marker for detection of bacterial infection. Similarly, the high affinity FcyRI (CD64), which is involved in the process of phagocytosis and intracellular killing of pathogens $(31,32)$, is also expressed at a very low level on the surface of

Table 1. Clinical characteristics of study population

\begin{tabular}{lccr}
\hline \multicolumn{1}{c}{ Characteristic } & Infected (group 1) & Noninfected (group 2) & Control (group 3) \\
\hline No. of infants $(n)$ & 32 & 58 & 20 \\
Gestational age (wk) & $29.2(27.8-31.2)$ & $28.6(27.1-30.1)$ & $29.1(27.5-31.2)$ \\
Birth weight (g) & $1183(864-1408)$ & $1131(903-1292)$ & $1180(845-1361)$ \\
Apgar scores & & & $7(6-8)$ \\
1 min & $7(5-8)$ & $6(4-8)$ & $9(8-9)$ \\
5 min & $9(8-9)$ & $9(7-9)$ & $10: 10$ \\
Male:female & $17: 15$ & $30: 28$ & \\
\hline
\end{tabular}

Results are median (interquartile range). 
Table 2. Details of pathologies and organisms of infected episodes $(\mathrm{n}=29) *$

\begin{tabular}{lcl}
\hline \multicolumn{1}{c}{ Organisms } & Episodes & \multicolumn{1}{c}{ Comments } \\
\hline Gram-positive organisms $[n=15(52 \%)]$ & & Septicemia $(\times 12) ;$ pneumonia $(\times 1)$ \\
Coagulase-negative Staphylococcus & 13 & Septicemia $(\times 1)$ \\
Staphylococcus aureus & 1 & Septicemia $(\times 1)$ \\
Enterococcus & 1 & Septicemia $(\times 2)$ \\
Gram-negative organisms $[n=6(21 \%)]$ & 2 & Septicemia $(\times 2) ;$ pneumonia $(\times 1)$ \\
Serratia marcescens & 3 & Septicemia $(\times 1)$ \\
Klebsiella sp. & 1 & Septicemia $(\times 2) ;$ meningitis $(\times 1)$ \\
Escherichia coli & & Septicemia $(\times 1)$ \\
Fungi $[n=4(13.5 \%)]$ & 3 & - \\
Candida albicans & 1 & 4 \\
Malassazia furfur & 4 & \\
\hline
\end{tabular}

* There were also eight cases of proven NEC of whom two had small bowel perforation.

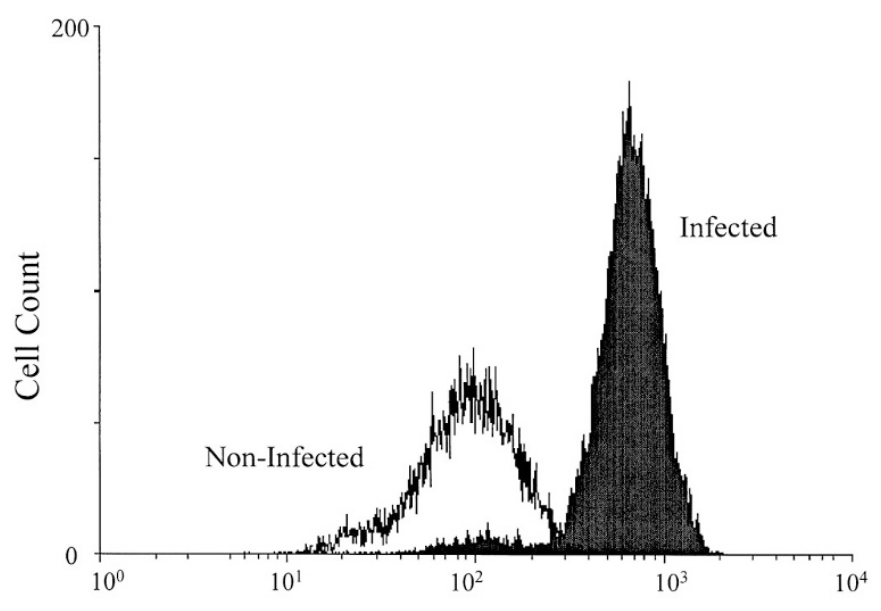

Fluorescence Intensity of CD64-PE on Neutrophils

Figure 1. Flow cytometric analysis of CD64 expression on neutrophils. Peripheral blood cells were stained with CD64-PE/CD45-perCP. The neutrophils were identified by the $\mathrm{CD} 45 /$ side-scatter profile. The graph represented a significant increase in CD64 expression in a typical infected neonate (solid histogram) when compared with a noninfected neonate (open histogram). The MFI values were transformed into PE-molecule bound/cell using the QuantiQuest computer software.

unstimulated neutrophils (18). During bacterial infection, the expression of CD64 on activated neutrophils was markedly increased $(18,33,34)$. More important, it has also been shown that neutrophils of preterm infants expressed CD64 antigen to an extent similar to those of older children and adults (18). Thus, this specific marker could potentially be used for identifying life-threatening infection in preterm VLBW infants.

In view of the high morbidity and mortality associated with late-onset nosocomial infection in VLBW infants $(1,2)$, a diagnostic test or a panel of tests with high sensitivity and negative predictive value are most desirable so that all positive cases can be correctly identified and treated (5). Moreover, to minimize the unnecessary use of antibiotics and misclassification of noninfected cases, the diagnostic test(s) should also have reasonably good specificity and positive predictive value (5). Although CD11b has been previously suggested to be a highly effective marker for diagnosing early-onset infection (infection occurs within $24 \mathrm{~h}$ of age) in newborn infants (19), our data did not support this notion. Its relatively low sensitivities (24-70\%) and specificities (71-75\%) made it unreli- able to be used as an infection marker for identifying late-onset nosocomial bacterial infection in VLBW infants. In addition, a recent study investigating more than 10 leukocyte surface markers was also unable to confirm the diagnostic value of $\mathrm{CD} 11 \mathrm{~b}$ for predicting infection in preterm infants (20). Several plausible explanations might have accounted for the discrepancy observed among different studies $(19,20)$. First, the populations being evaluated were very different. Both Weinschenk et al. (20) and the current study investigated only preterm (24-30 wk gestation) and VLBW infants, respectively, whereas the earlier study by Weirich et al. (19) involved mainly term infants. The expression of CD11b in neutrophils has been shown to be under developmental control, being down-regulated in preterm when compared with term infants and adults (35-37). The mobilization of CD11b by chemotactic and bacterial stimulation was also reported to be impaired in neonates (38). Thus, it is possible that the mechanism of $\mathrm{CD} 11 \mathrm{~b}$ activation in response to septic challenge may not be functionally mature or operational in some of our VLBW infants. Second, there were technical differences in the methods of measurement among different trials, and the number of PE-molecules bound per neutrophil was only quantitatively assessed in the present study. Moreover, as it has been reported that the purity of 1:1 antibody-PE conjugate (26) and the sensitivity of neutrophil CD11b expression to temperature (19) may affect the outcome of the quantitative analysis, these issues were addressed in this study by concurrently analyzing 67 infective episodes using the customized CD11b-PE conjugate of QuantiBRITE grade (Becton Dickinson Immunocytometry Systems) at a staining temperature of $4^{\circ} \mathrm{C}$. The sensitivities and specificities of the purified CD11b QuantiBRITE reagent ranged between 16 and $70 \%$ and 47 and $52 \%$ respectively, demonstrating that the modified procedures with purified reagent and lower temperature did not improve the diagnostic utilities of the CD11b test. Third, unlike early-onset infection in which the pathogens are usually acquired within a short and well-defined period during peripartum, one can never be certain in late-onset nosocomial sepsis at which phase of the infection blood was collected for determination of CD11b. Because activation of $\mathrm{CD} 11 \mathrm{~b}$ expression on neutrophils occurs early in the disease process (Fig. $2 A$ ), a delay in recognition of sepsis and consequently in obtaining the blood sample might result in missing the peak of CD11b expression. 
In contrast, CD64 exhibited high sensitivities (87-97\%) and specificities (88-90\%) during the study period (Table 4). Increase in neutrophil expression of CD64 was noted at the time of sepsis evaluation, and the level remained markedly raised at $24 \mathrm{~h}$ after the onset. CD64 positively identified all cases of septicemia, NEC, and CNS infection in this series.
A

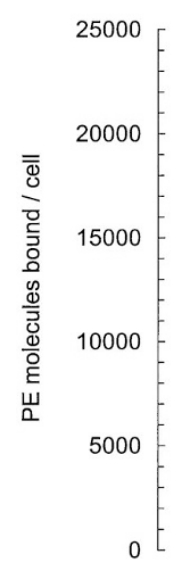

CD11b

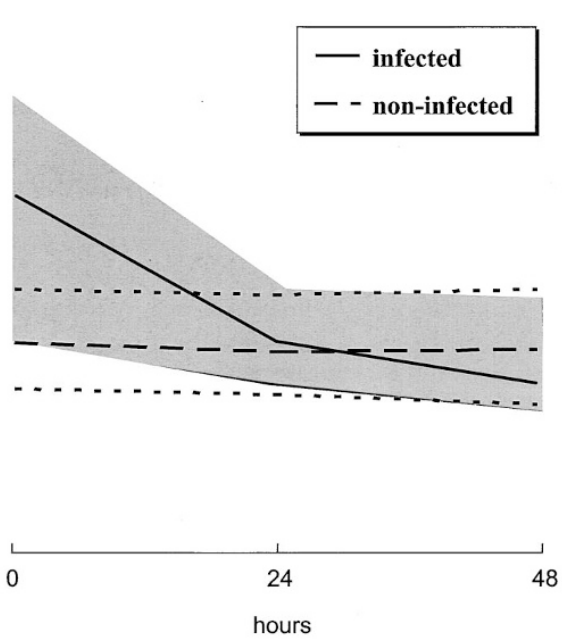

C

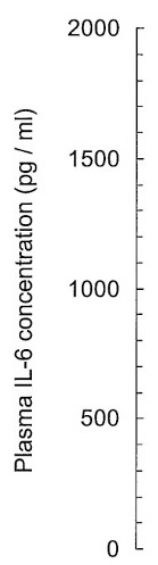

IL-6

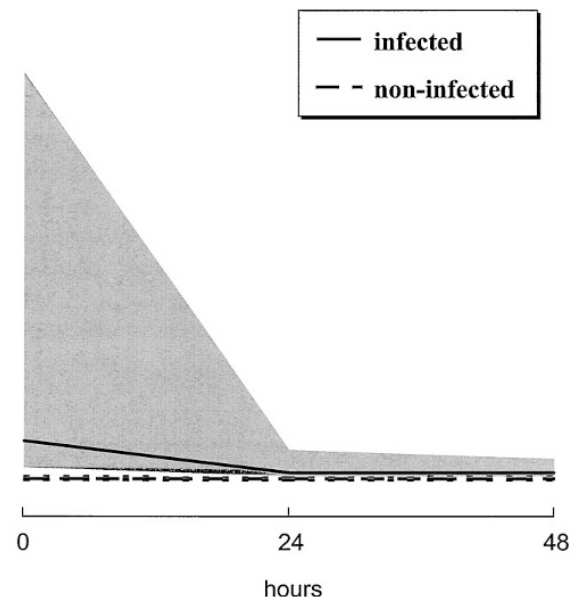

B
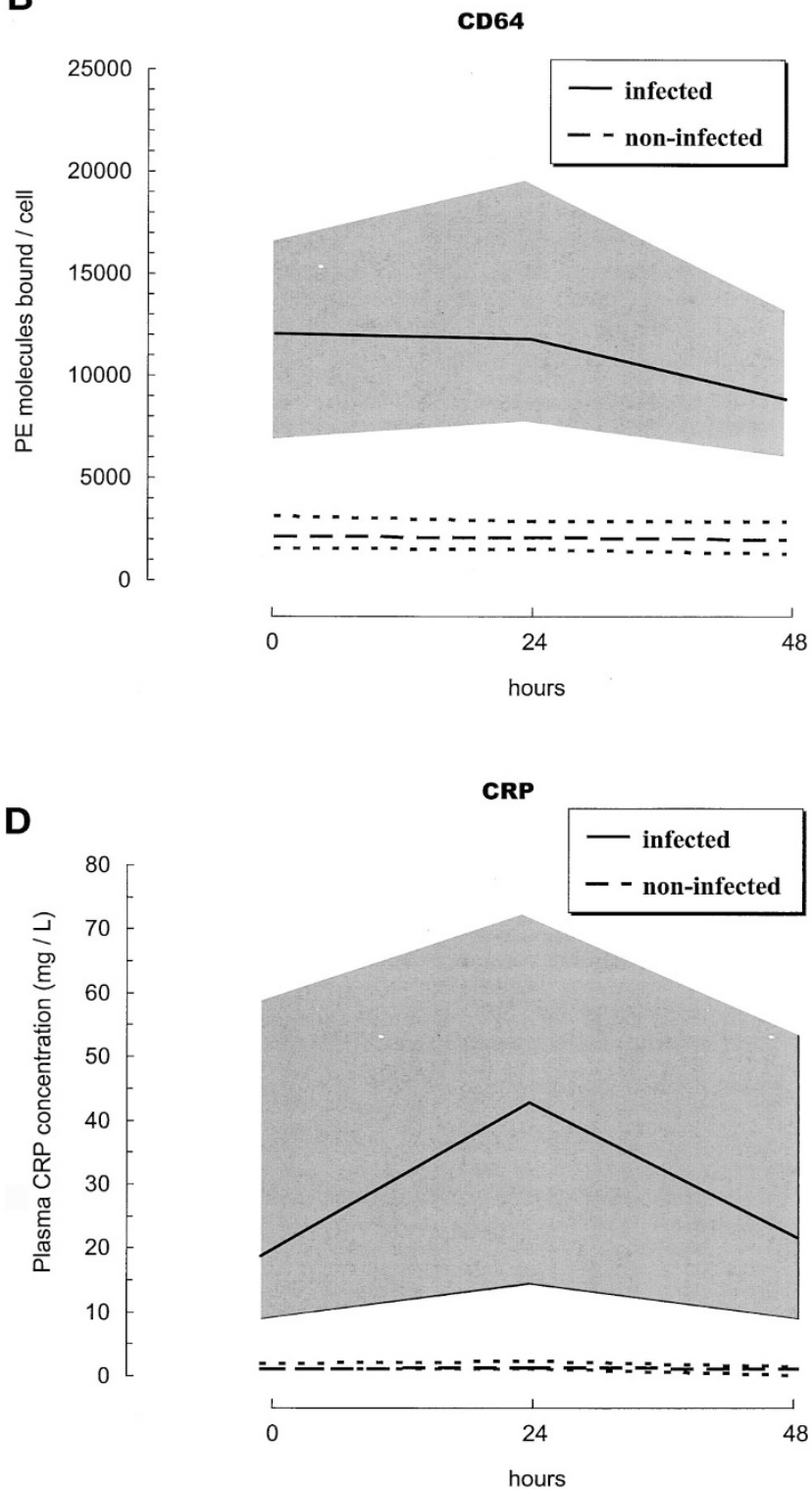

Figure 2. $A-D$, levels of the biochemical markers and neutrophil cell-surface antigen expression during the first $48 \mathrm{~h}$ of suspected clinical sepsis. Each figure compares the range of an infection marker between the infected and noninfected episodes. Data are expressed as median and 25th and 75th percentiles. Bold line and upper and lower boundaries of the shaded area represent the 50th, 75th, and 25th percentiles, respectively, of the infected episodes. Thick and thin dashed lines and their upper and the lower boundaries of the 50th, 75th, and 25th percentiles, respectively, of the noninfected episodes.

Table 3. Levels of biochemical markers and neutrophil cell-surface antigen expressions at onset (0 h) of sepsis evaluation

\begin{tabular}{lccr}
\hline \multicolumn{1}{c}{ Variable } & Infected (group 1) & Noninfected (group 2) & Control (group 3) \\
\hline $\begin{array}{l}\text { No. of episodes of suspected infection } \\
\quad \text { and no. of controls }(n)\end{array}$ & 37 & 90 & 20 \\
Postnatal age at blood sampling (d) & $27(16-52)$ & $21(14-36)$ & $21(17-25)$ \\
CD11b (PE-molecules bound/cell) & $* 15,549(8,514-19,354)$ & $8,526(6,324-10,982)$ & $8,699(6,482-10,177)$ \\
CD64 (PE-molecules bound/cell) & $* 11,995(6,934-15,382)$ & $2,154(1,585-3,120)$ & $1,611(1,116-2,890)$ \\
IL-6 $(\mathrm{pg} / \mathrm{mL})$ & $* 140(34-1,251)$ & $4(2-11)$ & $3(1-6)$ \\
CRP $(\mathrm{mg} / \mathrm{L})$ & $* 18(4-49)$ & $1(1-2)$ & $1(1-3)$ \\
\hline
\end{tabular}

Results are median (interquartile range).

* Levels of the four markers in the infected group are significantly increased when compared with the noninfected and control groups at $0 \mathrm{~h}(p<0.0001)$; no significant differences in the levels of the four markers are found between the noninfected and control groups. 
Table 4. Comparison of sensitivity, specificity, and positive and negative predictive values of markers using optimal cutoff values during first 48 h of suspected clinical sepsis

\begin{tabular}{|c|c|c|c|c|c|c|c|c|c|c|c|c|}
\hline \multirow{2}{*}{$\begin{array}{l}\text { Infection } \\
\text { markers }\end{array}$} & \multicolumn{4}{|c|}{$0 \mathrm{~h}$} & \multicolumn{4}{|c|}{$24 \mathrm{~h}$} & \multicolumn{4}{|c|}{$48 \mathrm{~h}$} \\
\hline & Sensitivity & Specificity & PPV & NPV & Sensitivity & Specificity & PPV & NPV & Sensitivity & Specificity & PPV & NPV \\
\hline CD11b & 0.70 & 0.72 & 0.50 & 0.86 & 0.25 & 0.71 & 0.30 & 0.70 & 0.24 & 0.75 & 0.30 & 0.70 \\
\hline CD64 & 0.95 & 0.88 & 0.80 & 0.97 & 0.97 & 0.90 & 0.80 & 0.99 & 0.87 & 0.86 & 0.80 & 0.93 \\
\hline IL-6 & 0.78 & 0.92 & 0.81 & 0.91 & 0.44 & 0.93 & 0.72 & 0.81 & 0.46 & 0.91 & 0.68 & 0.80 \\
\hline CRP & 0.65 & 0.99 & 0.96 & 0.87 & 0.72 & 1.00 & 1.00 & 0.90 & 0.65 & 1.00 & 1.00 & 0.87 \\
\hline
\end{tabular}

PPV, positive predictive value; NPV, negative predictive value.

\section{A 0 hour}

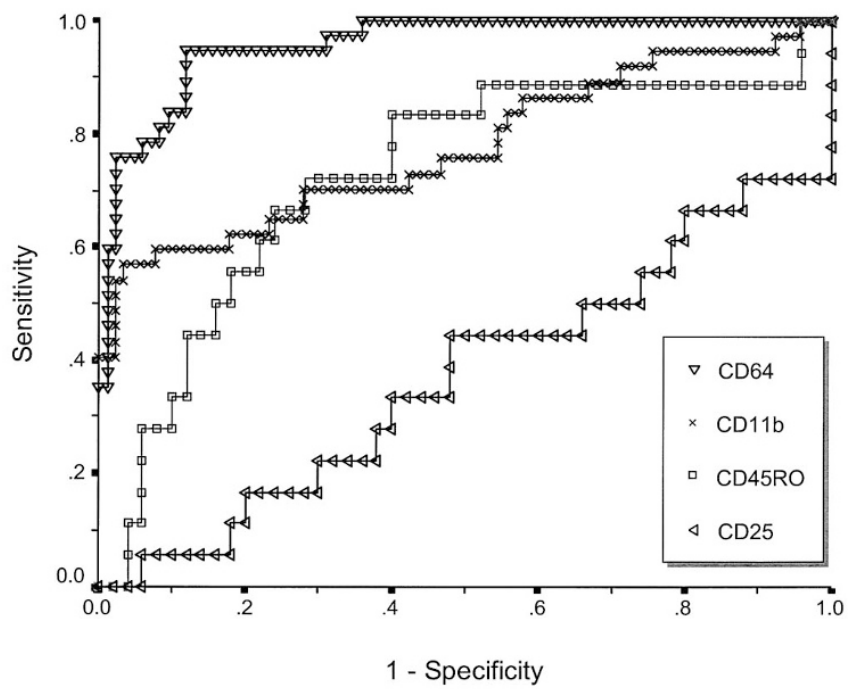

B 24 hours

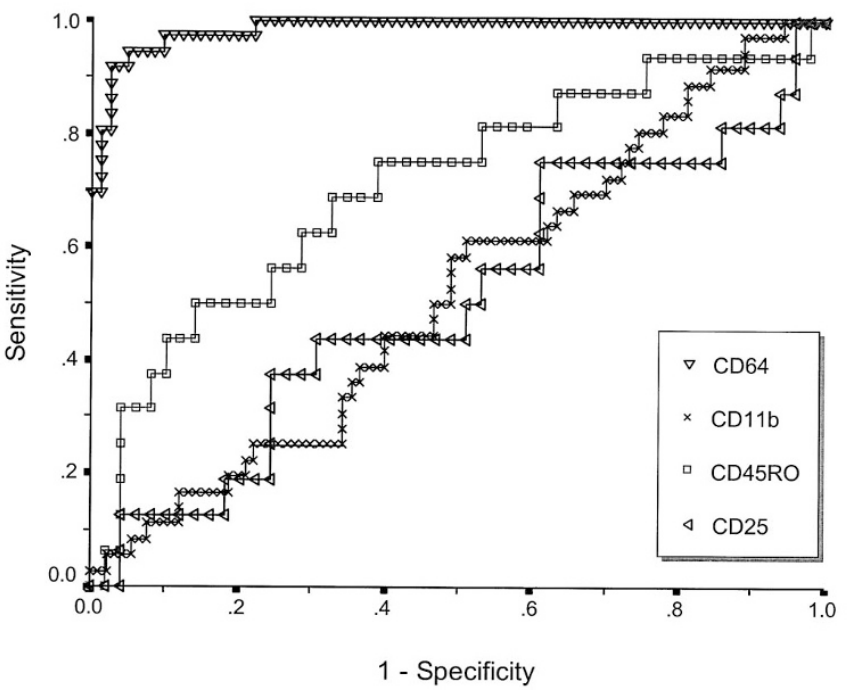

Figure 3. ROC of the leukocyte cell-surface antigens at $0(A)$ and $24 \mathrm{~h}(B)$.

Only one case of coagulase-negative Staphylococcus pneumonia was misclassified. Combining the use of IL- 6 or CRP at $0 \mathrm{~h}$ would further enhance the diagnostic capability for identifying serious localized chest infection, and improve the sensitivity and negative predictive value of CD64 to $100 \%$ (Table 5). Under such circumstances, approximately $10-14 \%$ of nonin- fected infants would erroneously be considered as septic and continue to receive antibiotic treatment unnecessarily. Also in accordance with previous findings (18), patients with Grampositive septicemia did not differ significantly from Gramnegative or fungal septicemia in their CD64 expression.

The four cases of infection in which no microorganisms could be isolated from the blood or CSF cultures, all presented with unstable temperature, hypotension, recurrent apnea, and severe desaturation. Two of the infants required intubation and were recommenced on assisted ventilation. These infants were judged to be genuinely septic on the basis of their strong and persistent signs of infection, and all cases were positively identified clinically before the results of the infection markers became available. Subsequently, these infants were found to have at least two abnormally raised markers of infection (CD64 and CRP \pm IL- 6 or CD11b). All received a full course of antibiotic treatment, and their progress of clinical improvement bore close temporal relation with the normalization of infection markers. Thus, we believed that these cases have not been misclassified.

Data concerning the use of cell-surface antigens as markers of infection in preterm infants are infrequently reported in the literature. To our knowledge, this study represents the first quantitative flow cytometric analysis of leukocyte cell-surface antigens for diagnosis of late-onset nosocomial infection in preterm VLBW infants. We have demonstrated that neutrophil expression of CD64 might be used as a sensitive diagnostic marker. However, CD11b, CD25, and CD45RO were found to be less useful as predictors of neonatal sepsis. Despite the very high sensitive and negative predictive value of CD64, most neonatal clinicians would still be reluctant to withhold antibiotic treatment for a deteriorating infant at the early phase of suspected clinical sepsis. However, the use of CD64 alone or in combination with other diagnostic markers such as IL-6 or CRP would allow clinicians to confidently discontinue antibiotics at $24 \mathrm{~h}$, without waiting for the definitive microbiologic results, in noninfected infants. Undoubtedly, the demand on the application of flow cytometry for cell-surface antigen detection, characterization of cell subpopulations, oncology, and other hematologic analyses will continue to grow, and will soon become an essential and integral part of routine laboratory investigations in most major hospitals and bone marrow transplant units. The quantitative flow cytometric analysis applied in this study could further be developed into a routine clinical test, with high comparability and reproducibility, across different laboratories. Measurement of neutrophil CD64 expression by this method is highly specific, and can be efficiently 
Table 5. Comparison of sensitivity, specificity, and positive and negative predictive values of different combinations of markers using optimal cutoff values within $48 \mathrm{~h}$ of onset of infection

\begin{tabular}{|c|c|c|c|c|c|c|}
\hline \multicolumn{3}{|c|}{ Combination of markers } & \multirow[b]{2}{*}{ Sensitivity } & \multirow[b]{2}{*}{ Specificity } & \multirow[b]{2}{*}{ PPV } & \multirow[b]{2}{*}{ NPV } \\
\hline $0 \mathrm{~h}$ & $24 \mathrm{~h}$ & $48 \mathrm{~h}$ & & & & \\
\hline CRP & CD64 & - & 1.00 & 0.90 & 0.80 & 1.00 \\
\hline- & CD64 & CRP & 0.97 & 0.91 & 0.82 & 0.99 \\
\hline - & CD64/CRP & - & 0.97 & 0.91 & 0.81 & 0.99 \\
\hline CD64 & CRP & - & 0.97 & 0.89 & 0.78 & 0.99 \\
\hline CD64 & - & CRP & 0.97 & 0.89 & 0.78 & 0.99 \\
\hline - & CD64 & IL-6 & 0.95 & 0.83 & 0.70 & 0.97 \\
\hline
\end{tabular}

PPV, positive predictive value; NPV, negative predictive value.

performed on an ad hoc basis using only minimal volume of blood sample $(0.05 \mathrm{~mL}$ of whole blood). Moreover, the result of the test can be made accessible within a few hours $(<4 \mathrm{~h})$ after the specimen reaches the laboratory. To further validate the value and applicability of CD64 for diagnosing lifethreatening infection in at-risk preterm and term infants, prospective studies examining different categories of infections (viral, bacterial, and fungal) and larger number of infants, including those with early-onset infection, should be performed.

Acknowledgment. The authors thank Melinda Leong, Becton Dickinson Immunocytometry Systems, Singapore, for her technical advice on this project.

\section{REFERENCES}

1. Stoll BJ, Gordon T, Korones SB, Shankaran S, Tyson JE, Bauer CR, Fanaroff AA, Lemons JA, Donovan EF, Oh W, Stevenson DK, Ehrenkranz RA, Papile LA, Verter J, Wright LL 1996 Late-onset sepsis in very low birth weight neonates: a report from the National Institute of Child Health and Human Development Neonatal Research Network. J Pediatr 129:63-71

2. Stoll BJ, Holman RC, Schuchat A 1998 Decline in sepsis-associated neonatal and infant deaths in the United States, 1979 through 1994. Pediatrics 102:e18; http:/ www.pediatrics.org/cgi/content/full/102/2e18

3. Rodwell RL, Leslie AL, Tudehope DI 1988 Early diagnosis of neonatal sepsis using a hematologic scoring system. J Pediatr 112:761-767

4. Berger C, Uehlinger J, Ghelfi D, Blau N, Fanconi S 1995 Comparison of C-reactive protein and white blood cell count with differential in neonates at risk of septicaemia. Eur J Pediatr 154:138-144

5. Ng PC, Cheng SH, Chui K, Fok TF, Wong MY, Wong W, Wong RPO, Cheung KL 1997 Diagnosis of late onset neonatal sepsis with cytokines, adhesion molecule, and C-reactive protein in preterm very low birthweight infants. Arch Dis Child 77:F221-F227

6. Wagle S, Grauaug A, Kohan R, Evans SF 1994 C-reactive protein as a diagnostic tool of sepsis in very immature babies. J Paediatr Child Health 30:40-44

7. Franz AR, Steinbach G, Kron M, Pohlandt F 1999 Reduction of unnecessary antibiotic therapy in newborn infants using interleukin-8 and C-reactive protein as markers of bacterial infections. Pediatrics 104:447-453

8. Hatherill M, Tibby SM, Sykes K, Turner C, Murdoch IA 1999 Diagnostic markers of infection: comparison of procalcitonin with $\mathrm{C}$ reactive protein and leucocyte count. Arch Dis Child 81:417-421

9. Gendrel D, Assicot M, Raymond J, Moulin F, Francoual C, Badoual J, Bohuon C 1996 Procalcitonin as a marker for the early diagnosis of neonatal infection. J Pediatr 128:570-573

10. Assicot M, Gendrel D, Carsin H, Raymond J, Guilband J, Bohuon C 1993 High serum procalcitonin concentrations in patients with sepsis and infection. Lancet 341:515-518

11. Messer J, Eyer D, Donato L, Gallati H, Matis J, Simeoni U 1996 Evaluation of interleukin-6 and soluble receptors of tumor necrosis factor for early diagnosis of neonatal infection. J Pediatr 129:574-580

12. Küster H, Weiss M, Willeitner AE, Detlefsen S, Jeremias I, Zbojan J, Geiger R, Lipowsky G, Simbruner G 1998 Interleukin-1 receptor antagonist and interleukin-6 for early diagnosis of neonatal sepsis 2 days before clinical manifestation. Lancet $352: 1271-1277$

13. Jurges ES, Henderson DC 1996 Inflammatory and immunological markers in preterm infants: correlation with disease. Clin Exp Immunol 105:551-555

14. Berner R, Tüxen B, Clad A, Forster J, Brandis M 2000 Elevated gene expression of interleukin-8 in cord blood is a sensitive marker for neonatal infection. Eur J Pediatr 159:205-210
15. van der Poll T, Malefyt R, Coyle SM, Lowry SF 1997 Antiinflammatory cytokine responses during clinical sepsis and experimental endotoxemia: sequential measurements of plasma soluble interleukin (IL)-1 receptor type II, IL-10, and IL-13. J Infect Dis $175: 118-122$

16. Zilow G, Zilow EP, Burger R, Linderkamp O 1993 Complement activation in newborn infants with early onset infection. Pediatr Res 34:194-203

17. Guillois B, Donnou MD, Sizun J, Bendaoud B, Youinon P 1994 Comparative study of four tests of bacterial infection in the neonate. Biol Neonate 66:175-181

18. Fjaertoft G, Håkansson L, Ewald U, Foucard T, Venge P 1999 Neutrophils from term and preterm newborn infants express the high affinity Fc $\gamma$-receptor I (CD64) during bacterial infection. Pediatr Res 45:871-876

19. Weirich E, Rabin RL, Maldonado Y, Benitz W, Modler S, Herzenberg LA, Herzenberg LA 1998 Neutrophil CD11b expression as a diagnostic marker for early-onset neonatal infection. J Pediatr 132:445-451

20. Weinschenk NP, Farina A, Bianchi DW 2000 Premature infants respond to earlyonset and late-onset sepsis with leukocyte activation. J Pediatr 137:345-350

21. Brüning T, Daiminger A, Ender G 1997 Diagnostic value of CD45RO expression on circulating $\mathrm{T}$ lymphocytes of fetuses and newborn infants with pre-, peri- or early post-natal infections. Clin Exp Immunol 107:306-311

22. Hodge S, Hodge G, Flower R, Han P 1998 Surface activation markers of $T$ lymphocytes: role in the detection of infection in neonates. Clin Exp Immunol 113:33-38

23. Michie C, Harvey D 1994 Can expression of CD45RO, a T-cell surface molecule, be used to detect congenital infection? Lancet 343:1259-1260

24. Walsh MC, Kliegman RM, Fanaroff AA 1988 Necrotizing enterocolitis: a practitioner's prospective. Pediatr Rev 9:219-226

25. Iyer SB, Hultin LE, Zawadzki JA, Davis KA, Giorgi JV 1998 Quantitation of CD38 expression using QuantiBRITE beads. Cytometry 33:206-212

26. Gratama JW, D'hautcourt J-L, Mandy F, Rothe G, Barnett D, Janossy G, Papa S, Schmitz G, Lenkei R 1998 Flow cytometric quantitation of immunofluorescence intensity: problems and perspectives. Cytometry 33:166-178

27. Goldstein H 1995 Multilevel Statistical Models. Edward Arnold, London, pp 87-94

28. Hedeker D, Gibbons RD 1996 MIXREG: a computer program for mixed-effects regression analysis with autocorrelated errors. Comput Methods Programs Biomed $49 \cdot 229-252$

29. Lehr HA, Krombach F, Munzing S, Bodlaj R, Glaubitt SI, Seiffge D, Hubner C, von Andrian UH, Messmer K 1995 In vitro effects of oxidized low density lipoprotein on $\mathrm{CD} 11 \mathrm{~b} / \mathrm{CD} 18$ and $\mathrm{L}$-selectin presentation on neutrophils and monocytes with relevance for the in vivo situation. Am J Pathol 146:218-227

30. Simms HH, D'Amico R 1995 Lipopolysaccharide induces intracytoplasmic migration of the polymorphonuclear leukocyte CD11b/CD18 receptor. Shock 3:196-203

31. de Haas M, Vossebeld PJM, Von dem Borne AEGK, Roos D $1995 \mathrm{Fc} \gamma$ receptors of phagocytes. J Lab Clin Med 126:330-341

32. Sanchez-Mejorada G, Rosales C 1998 Signal transduction by immunoglobulin Fc receptors. J Leukoc Biol 63:521-533

33. Herra CM, Keane CT, Whelan A 1996 Increased expression of Fc $\gamma$ receptors on neutrophils and monocytes may reflect ongoing bacterial infection. J Med Microbiol 44:135-140

34. Leino L, Sorvajärvi K, Katajistos J, Laine M, Lilius E-M, Pelliniemi TT, Rajamaki A, Silvoniemi P, Nikoskelainen J 1997 Febrile infection changes the expression of IgG $\mathrm{Fc}$ receptors and complement receptors in human neutrophils in vivo. Clin Exp Immunol 107:37-43

35. McEvoy LT, Zakem-Cloud H, Tosi MF 1996 Total cell content of CR3 (CD11b CD18) and LFA-1 (CD11a/CD18) in neonatal neutrophils: relationship to gestational age. Blood 87:3929-3933

36. Abughali N, Berger M, Tosi MF 1994 Deficient total cell content of CR3 (CD11b) in neonatal neutrophils. Blood 83:1086-1092

37. Kaufman D, Kilpatrick L, Hudson RG, Campbell DE, Kaufman A, Douglas SD, Harris M 1999 Decreased superoxide production, degranulation, tumor necrosis factor alpha secretion, and $\mathrm{CD} 11 \mathrm{~b} / \mathrm{CD} 18$ receptor expression by adherent monocytes from preterm infants. Clin Diagn Lab Immunol 6:525-529

38. Török C, Lundahl JH, Lagercrentz H 1993 Diversity in regulation of adhesion molecules (Mac-1 and L-selectin) in monocytes and neutrophils from neonates and adults. Arch Dis Child 68:561-565 